METHODOLOGICAL NOTES

Diffraction radiation from a charge as radiation from a superluminal source in a vacuum

To cite this article: A P Potylitsyn et al 2020 Phys.-Usp. 63303

View the article online for updates and enhancements. 


\title{
Diffraction radiation from a charge as radiation from a superluminal source in a vacuum
}

\author{
A P Potylitsyn, D Yu Sergeeva, M N Strikhanov, A A Tishchenko
}

DOI: https://doi.org/10.3367/UFNe.2019.10.038667

\section{Contents}

1. Introduction

303

2. Diffraction radiation as Cherenkov radiation

303

3. Diffraction radiation of a charge for arbitrary geometry

305

4. Coherent diffraction radiation from short electron bunches

306

5. Threshold condition for the occurrence of 'superluminal' Cherenkov radiation in a vacuum

307

6. Conclusions

References

308

308

Abstract. An analysis of spectral-angular characteristics of diffraction radiation, both incoherent and coherent, has been performed. It is shown that radiation processes can be interpreted as Cherenkov radiation, which is produced by a region of dynamic polarization moving along a target edge with superluminal velocity $v_{\mathrm{SL}}$. Such radiation is generated if the condition $v_{\mathrm{SL}}>\boldsymbol{c}$ is fulfilled, which is the conventional 'threshold' Cherenkov condition.

Keywords: diffraction radiation, transition radiation, superluminal source, beam diagnostics, $\mathrm{THz}$ radiation

\section{Introduction}

Characteristics of Cherenkov Radiation (CR) accompanying the motion of a superluminal source in a vacuum and possible realizations of such sources are thoroughly analyzed by the authors of Refs [1-3].

Reference [4] considered radiation from a charged particle crossing a dielectric fiber and showed that for small angles $\psi_{0}$ between the particle trajectory and the fiber the radiation angular distribution is concentrated along generating lines of a cone with an axis that coincides with the fiber and the angle of $\theta_{\text {ch }}=\psi_{0}$ to the axis. This radiation was described in terms of transition radiation (TR); however, with the same success, it could be interpreted as CR whose source is a perturbation

A P Potylitsyn ${ }^{(1,2, *)}$, D Yu Sergeeva ${ }^{(2,3,4)}$,

M N Strikhanov ${ }^{(2)}$, A A Tishchenko ${ }^{(2,3,4)}$

(1) National Research Tomsk Polytechnic University, prosp. Lenina 30, 634050 Tomsk, Russian Federation

(2) National Research Nuclear University MEPhI,

Kashirskoe shosse 31, 115409 Moscow, Russian Federation

(3) National Research Center 'Kurchatov Institute',

pl. Akademika Kurchatova 1, 123182 Moscow, Russian Federation

(4) Laboratory of Radiation Physics, Belgorod State National Research

University, ul. Pobedy 85, 308015 Belgorod, Russian Federation

E-mail: ${ }^{(*)}$ potylitsyn@tpu.ru

Received 24 April 2019, revised 1 October 2019

Uspekhi Fizicheskikh Nauk 190 (3) 329-335 (2020)

Translated by S D Danilov; edited by V L Derbov to electron shells of a target material moving along the fiber at a superluminal velocity.

In the subsequent study [5] the authors showed that the diffraction radiation (DR) arising when a charge passes by a fiber-like target can also be interpreted as CR from a superluminal source. For the orientation angles $\psi_{0} \sim \gamma^{-1}$ (where $\gamma$ is the Lorentz factor of a charged particle), the radiation is confined along cone generating lines and proves to be nearly azimuthally isotropic, as one would expect for a symmetric arrangement.

References [6, 7] show that the process of coherent transition radiation from an electron bunch (TR with a wavelength $\lambda$ smaller than the bunch size) in some cases can be considered superluminal CR generated by the intersection domain of the bunch and an oblique target moving at superluminal speed. In this case, as mentioned in Ref. [3], the effect "is that the radiating domain moves at a superluminal speed, but each pulse of radiation is caused by a new particle." Of course, what is meant is not the particles in the bunch, but those in the target - atoms, molecules, whose contribution to the radiation process is synchronized by the field of a passing charged particle. As mentioned by V L Ginzburg [2], it is exactly the presence of many particles participating in the process that eliminates the contradiction with the proposition of special relativity that the speed of light in a vacuum is the highest signal propagation speed.

In this paper, we consider the characteristics of superluminal CR induced by the interaction of a Coulomb field of charged particles with target atoms, namely diffraction radiation [8-10], leaving beyond the scope such exotic superluminal sources as light spots moving along the target surface $[11,12]$. We explore the characteristics of DR from an ideally conducting target for arbitrary angles of orientation between particle momenta and the target, i.e., for geometries that are of interest to experimenters.

\section{Diffraction radiation as Cherenkov radiation}

The term diffraction radiation refers to the radiation arising when a charge passes close to a target. Before considering radiation from bunches, we concentrate on diffraction 
radiation from a single particle when impact parameter $h$ (the minimum distance between the particle trajectory and the target edge) satisfies the condition

$$
h \leqslant \gamma \lambda,
$$

where $\lambda$ is the radiation wavelength. This inequality is dictated by the details of spectral distribution pertaining to diffraction radiation.

In metals, the approximation of ideal conductivity is applicable with a high accuracy; in this approximation, the spectrum of diffraction radiation, similar to the spectrum of transition radiation, is independent of frequency over a wide frequency range, from zero to ultraviolet frequencies. In the $\mathrm{X}$-ray range, the response for all materials, including metals, is described by the universal asymptotics of permittivity (see, e.g., $[13, \S 78])$. For transition radiation, this implies that the maximum frequency is defined by the product $\gamma \omega_{\mathrm{p}}$; the quantity $\omega_{\mathrm{p}}$ is called a plasma frequency in the physics of charged particle radiation (it should be distinguished from the Langmuir frequency which, not infrequently, is also called a plasma frequency in optics). In diffraction radiation, a new element appears, absent from transition radiation: an exponential decay with the maximum magnitude of the argument determined by the decay of the Fourier transform of the moving charge field in the direction transverse to its motion,

$$
\exp \left(-\frac{2 \omega h}{\gamma \beta c}\right)
$$

Thus, the maximum frequency $\omega_{\mathrm{c}}$ (the spectral cutoff frequency) of diffraction radiation is the minimum of the two values

$$
\omega_{\mathrm{c}}=\min \left\{\frac{\gamma \beta c}{2 h}, \gamma \omega_{\mathrm{p}}\right\} .
$$

The impact parameter $h$ is bounded by the beam transverse size, which for existing setups is not less than $10 \mu \mathrm{m}$. Thus, in practice, the inequality

$$
\omega_{\mathrm{p}} \gg \frac{\beta c}{2 h}
$$

commonly holds, so that the main role in the spectrum of diffraction radiation is played by the magnitude of the impact parameter, which makes the diffraction radiation distinct from the transition one.

Figure 1a depicts a typical geometry of diffraction radiation when the charge velocity vector is oriented at some angle to the target edge. As shown in Refs [14, 15], DR in such a geometry is emitted in the form of a cone, characteristic of $\mathrm{CR}$, with the opening half-angle $\theta_{\text {ch }}$ determined by the angle between particle's momentum and target edge (the $y$ axis in Fig. 1a).

We note that often, by analogy with transition radiation, it is supposed that the DR in the relativistic case is generated in the form of two branches: 'forward' DR (FDR) and 'backward' DR (BDR), the first being emitted in the direction of charge velocity, and the second one in the direction of mirror reflection [16] (Fig. 1b). The angle $\psi_{\mathrm{m}}$ corresponding to the maximum intensity of FDR and BDR, with an accuracy up to $\gamma^{-2}$, coincides with the angle $\psi_{0}$ between the particle's momentum and the target edge.
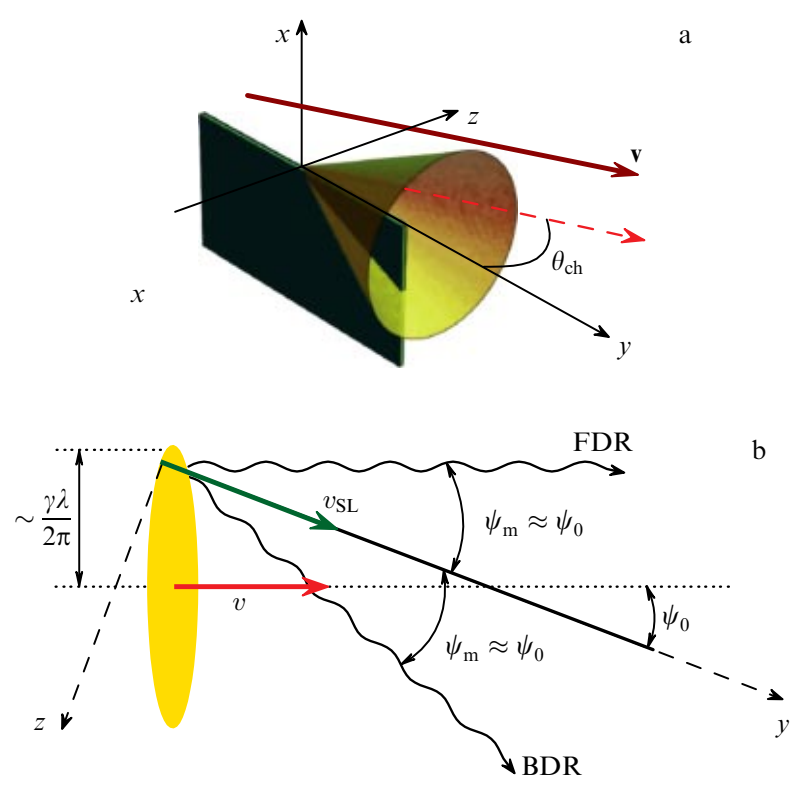

Figure 1. (a) Layout of diffraction radiation generation and the cone of Cherenkov radiation; (b) two branches of diffraction radiation (FDR and BDR) propagating along the cone generating lines.

However, as can be readily seen by comparing Figs 1a and $\mathrm{b}$, it is a cone that is emitted in reality, and the just mentioned forward and backward directions are nothing more than two generating lines seen in a section of the cone by a plane, where radiation is maximal.

DR is generated in the region close to the target edge crossed by the Coulomb field of the charge. For an ultrarelativistic charge, the Coulomb field is disk-shaped with an effective radius of $\gamma \lambda /(2 \pi)$. For an 'oblique' crossing of the target edge, the region excited by the particle field moves along the target edge with the velocity $v_{\mathrm{SL}}$ determined from elementary relationships

$$
v_{\mathrm{SL}}=\frac{2 \gamma \lambda}{2 \pi \Delta t \sin \psi_{0}}, \quad \Delta t=\frac{2 \gamma \lambda}{2 \pi \beta c \tan \psi_{0}},
$$

where $v=\beta c$ is the charge velocity.

As follows from (5), the velocity of radiation source $v_{\mathrm{SL}}$ depends on the charge velocity and the angle the target is inclined, and to realize the CR mechanism the condition

$$
v_{\mathrm{SL}} \equiv \frac{\beta c}{\cos \psi_{0}}>c
$$

needs to be satisfied, whence follows the 'threshold' condition for the appearance of CR,

$$
\beta>\cos \psi_{0}
$$

and the relationship defining the opening of the CR cone,

$$
\cos \theta_{\mathrm{ch}}=\frac{c}{v_{\mathrm{SL}}}=\frac{\cos \psi_{0}}{\beta} .
$$

In the ultra-relativistic approximation, Eqn (8), with accuracy up to terms of the order of $\gamma^{-2}$, leads to a relationship between the cone opening half-angle and target orientation

$$
\theta_{\mathrm{ch}}=\psi_{0},
$$


i.e., the 'superluminal' source with the velocity $v_{\mathrm{SL}}$, directed along the target edge, generates $\mathrm{CR}$ along generating lines of a cone with the opening half-angle $\psi_{0}$ (Fig. 1b). From this standpoint, the DR angular distribution, comprising both the FDR and BDR, can be considered a manifestation of 'superluminal' CR.

\section{Diffraction radiation of a charge for arbitrary geometry}

For an perfectly-conducting target, relationship (8) can be derived from a rigorous DR model.

Figure 2 shows DR generation for a target oriented arbitrarily relative to the velocity of a charged particle. The following geometry is used further: the $z$ axis is directed perpendicular to the target surface, the $y$ axis is along the target edge, $\psi$ is the angle between the wave vector and the $y$ axis, and $\varphi$ is the azimuth angle. The DR characteristics are governed by three components of the velocity vector:

$$
\begin{aligned}
\mathbf{v} & =c \boldsymbol{\beta}=c\left\{\beta_{x}, \beta_{y}, \beta_{z}\right\} \\
& =c \beta\left\{\sin \psi_{0} \cos \varphi_{0}, \cos \psi_{0}, \sin \psi_{0} \sin \varphi_{0}\right\},
\end{aligned}
$$

where $\psi_{0}$ is the angle between the velocity vector and the $y$ axis, and $\varphi_{0}$ is the velocity vector azimuth angle. The DR spectral-angular distribution is given by the following formula (see expression (3.76) in book [16]):

$$
\begin{aligned}
& \frac{\mathrm{d}^{3} W}{\hbar \mathrm{d} \omega \mathrm{d} \psi \mathrm{d} \varphi}=\frac{\alpha}{4 \pi^{2}} \beta_{\perp}\left(1-\beta_{y} \cos \psi\right) \\
& \quad \times \exp \left(-\frac{4 \pi h}{\lambda \beta_{\perp}} \sqrt{\left(1-\beta_{y} \cos \psi\right)^{2}-\beta_{\perp}^{2} \sin ^{2} \psi}\right) \\
& \quad \times \frac{1}{\left(1-\beta_{y} \cos \psi\right)^{2}-\beta_{\perp}^{2} \sin ^{2} \psi} \\
& \quad \times\left[\left(1-\beta_{y} \cos \psi-\beta_{x} \sin \psi \cos \varphi\right)^{2}-\beta_{z}^{2} \sin ^{2} \psi \sin ^{2} \varphi\right]^{-1} \\
& \quad \times\left\{\left[\left(1-\beta_{y} \cos \psi\right)^{2}-\beta_{\perp}^{2} \sin ^{2} \psi\right]\right. \\
& \quad \times\left(1+\frac{\beta_{x} \sin \psi}{1-\beta_{y} \cos \psi}\right)(1-\cos \varphi)+\left(\cos \psi-\beta_{y}\right)^{2} \\
& \left.\quad \times\left(1-\frac{\beta_{x} \sin \psi}{1-\beta_{y} \cos \psi}\right)(1+\cos \varphi)\right\}
\end{aligned}
$$

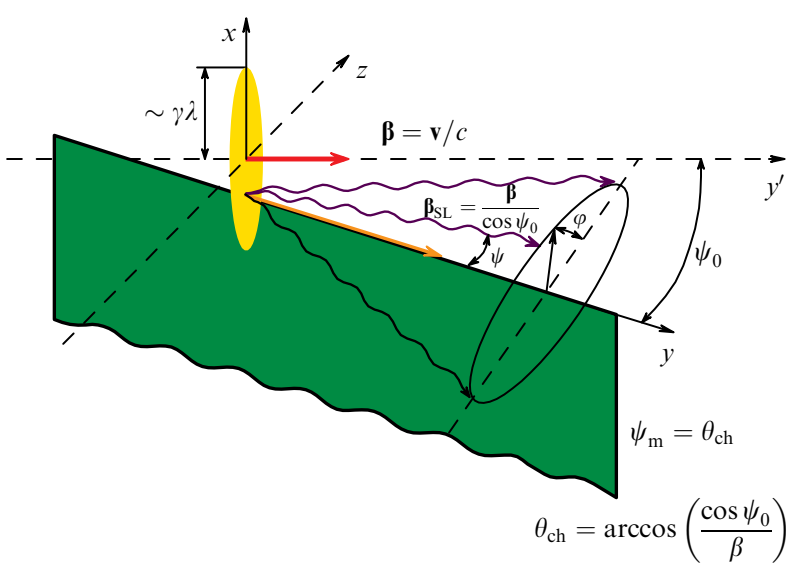

Figure 2. Geometry and angular variables for the description of the DR process.

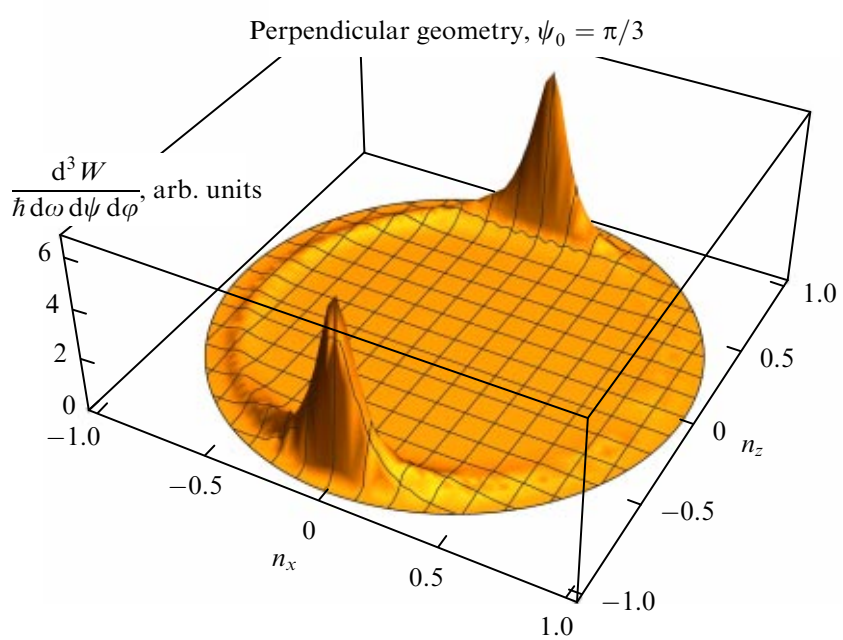

Figure 3. Angular distribution of DR intensity for the perpendicular geometry, the target inclination angle relative to electron velocity being $\psi_{0}=\pi / 3$. The other parameters: $\gamma=10, h=1 \mathrm{~mm}, \lambda=0.5 \mathrm{~mm}$.

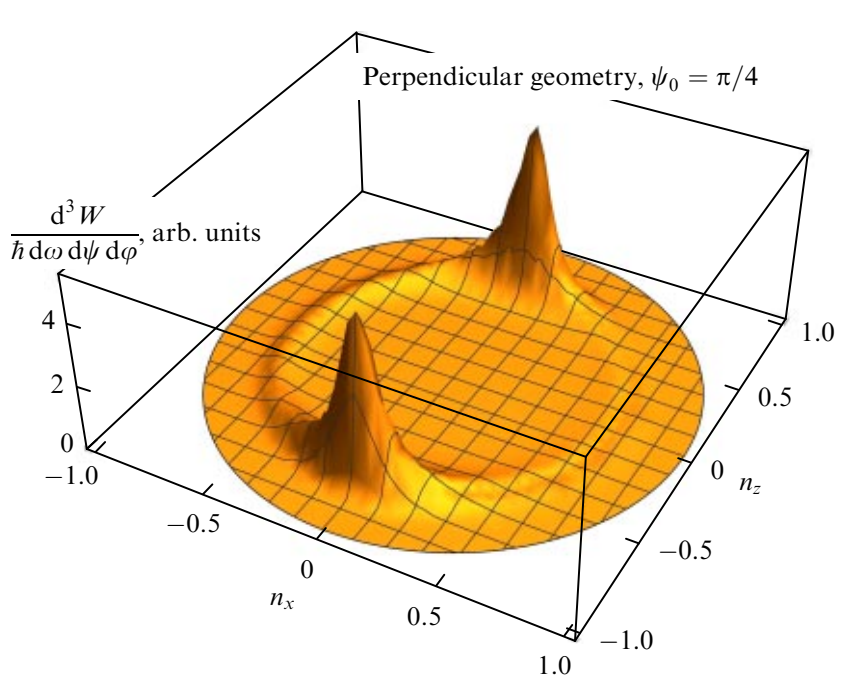

Figure 4. The same as in Fig. 3, but for the angle $\psi_{0}=\pi / 4$.

where $\alpha=1 / 137$ is the fine structure constant and $\beta_{\perp}=$ $\left(\beta_{x}^{2}+\beta_{z}^{2}\right)^{1 / 2}$.

For clarity, we consider two particular geometries: perpendicular $\left(\varphi_{0}=\pi / 2\right.$, i.e., $\left.\beta_{x}=0\right)$ and parallel $\left(\varphi_{0}=0\right.$, i.e., $\left.\beta_{z}=0\right)$.

Figures 3 and 4 show angular DR distributions in the perpendicular geometry for the following conditions:

$$
\gamma=10, \quad h=1 \mathrm{~mm}, \quad \lambda=0.5 \mathrm{~mm}, \quad \varphi_{0}=90^{\circ},
$$

and for the inclination angles $\psi_{0}=\pi / 3$ and $\psi_{0}=\pi / 4$. For better readability, the DR distributions are given as functions of directional cosines:

$$
\begin{aligned}
& n_{x}=\sin \psi \cos \varphi, \\
& n_{y}=\cos \psi, \\
& n_{z}=\sin \psi \sin \varphi .
\end{aligned}
$$

Analogous distributions for the parallel $\left(\varphi_{0}=0\right)$ geometry are plotted in Figs 5 and 6 . It can be clearly seen from the figures that the DR intensity attains a maximum for the polar 


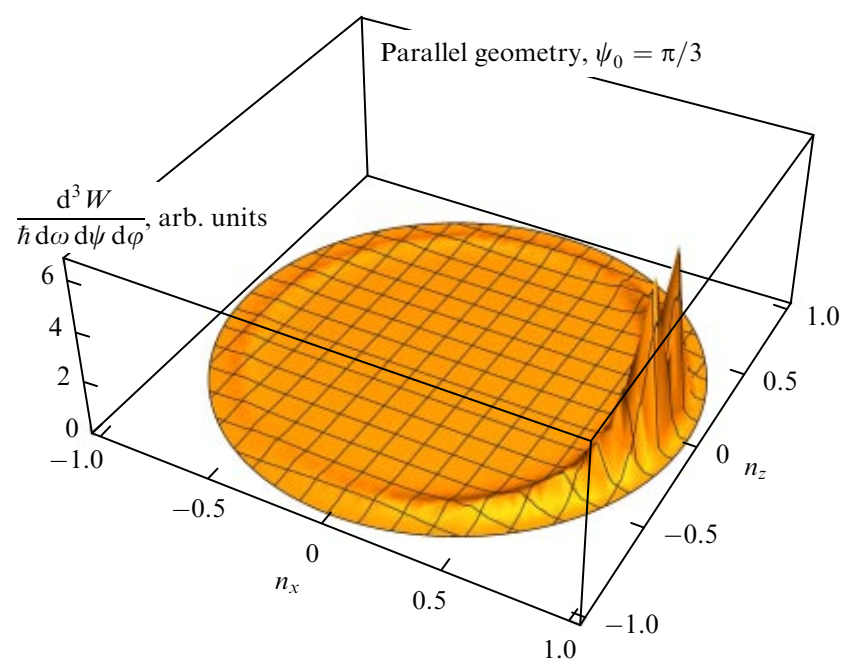

Figure 5. The same as in Fig. 3, but for the parallel geometry and the angle $\psi_{0}=\pi / 3$.

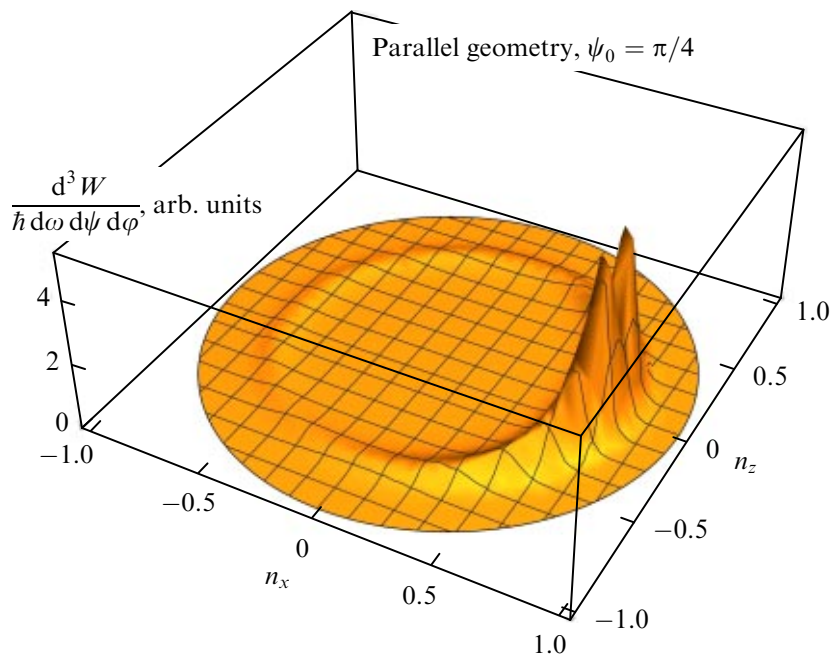

Figure 6. The same as in Fig. 5, but for the angle $\psi_{0}=\pi / 4$.

angle of $\psi_{\mathrm{m}}$ :

$$
\psi_{\mathrm{m}} \approx \psi_{0}, \quad \psi_{0}=\frac{\pi}{3}, \frac{\pi}{4},
$$

i.e., along the conical surface with the apex angle $\psi_{0}$.

Relationship (8) obtained earlier from qualitative considerations can be derived from rigorous formula (10). As shown in Ref. [15], the DR angular distribution is mainly defined by the exponential factor in formula (10). It can be readily seen that for the perpendicular geometry $\left(\beta_{x}=0\right)$ the argument of this exponential function attains the minimum value $-4 \pi h /(\gamma \beta \lambda)$ provided the condition

$$
\cos \psi=\beta^{-1} \cos \psi_{0}
$$

which, with account for variables (11), takes the form

$$
n_{y}=\beta^{-1} \cos \psi_{0}=\frac{\beta_{y}}{\beta^{2}},
$$

which is identical to relationship (8) derived from qualitative considerations. Condition (12b) was already obtained as early as in Ref. [9]; however, only Refs [14, 15] found that it leads to a so-called conical effect in diffraction radiation and SmithPurcell radiation.

\section{Coherent diffraction radiation from short electron bunches}

Angular characteristics of coherent diffraction radiation generated by an electron bunch with longitudinal size much larger than the transverse one do not differ from those given in Figs 3-6.

Recent laser-plasma acceleration technologies allow creating and accelerating electron bunches with longitudinal sizes much smaller than the transversal ones (see, for example, [17-19]). Coherent diffraction radiation from such bunches can be considered by analogy with the coherent transition radiation of similar bunches [7] if their transverse size is much smaller than the impact parameter $h$. In this case, the characteristics of radiation are determined by the form factor $F(\lambda, \mathbf{n})$ :

$$
\frac{\mathrm{d}^{2} W_{\mathrm{CDR}}}{\hbar \mathrm{d} \omega \mathrm{d} \Omega}=N_{\mathrm{e}}\left(1+F(\lambda, \mathbf{n}) N_{\mathrm{e}}\right) \frac{\mathrm{d}^{2} W}{\hbar \mathrm{d} \omega \mathrm{d} \Omega},
$$

where $N_{\mathrm{e}}$ is the number of particles in the bunch.

The form factor is commonly expressed in terms of the charge distribution in the bunch $\rho(x, y, z)$ :

$$
F(\lambda, \mathbf{n})=\left|\int \mathrm{d} \mathbf{r} \rho(\mathbf{r}) \exp (-\mathrm{i} \Delta \varphi)\right|^{2},
$$

where $\Delta \varphi$ is the phase factor, which, for the DR, by analogy with the TR [7], can be written in the form

$$
\Delta \varphi=\frac{2 \pi}{\lambda}\left(-y n_{y}^{\prime}+\frac{z^{\prime}}{\beta}\right), \quad n_{y}^{\prime}=\frac{n_{y}-\beta^{-1} \cos \psi_{0}}{\sin \psi_{0}} .
$$

The phase factor (15) for the bunch depends on both the coordinate $y$ (along the edge) and the distance $z$ between the bunch electrons.

Different from the single charge diffraction radiation considered earlier, with the characteristics of superluminal CR governed by the charge velocity $\beta c$ and target inclination angle $\psi_{0}$, the characteristics of coherent diffraction radiation from ultrashort bunches also depend on the angle $\chi_{0}$ between the minor axis of the ellipsoid describing the charge distribution in the bunch and its velocity (Fig. 7).

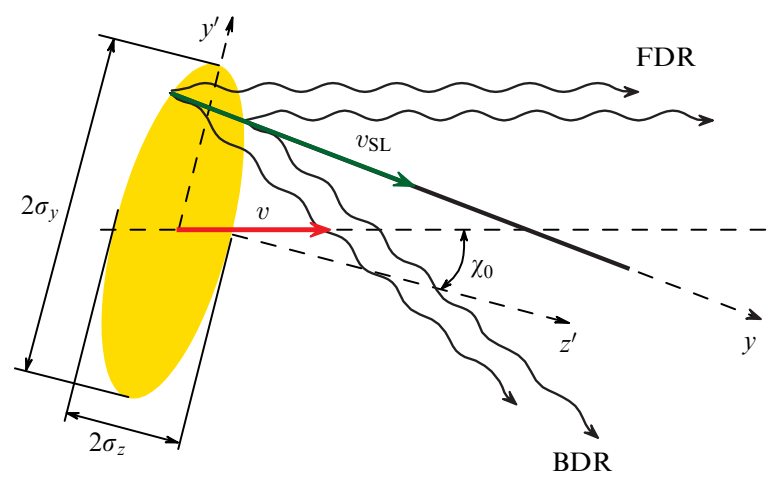

Figure 7. Coherent DR generation by an ultrashort electron bunch. 


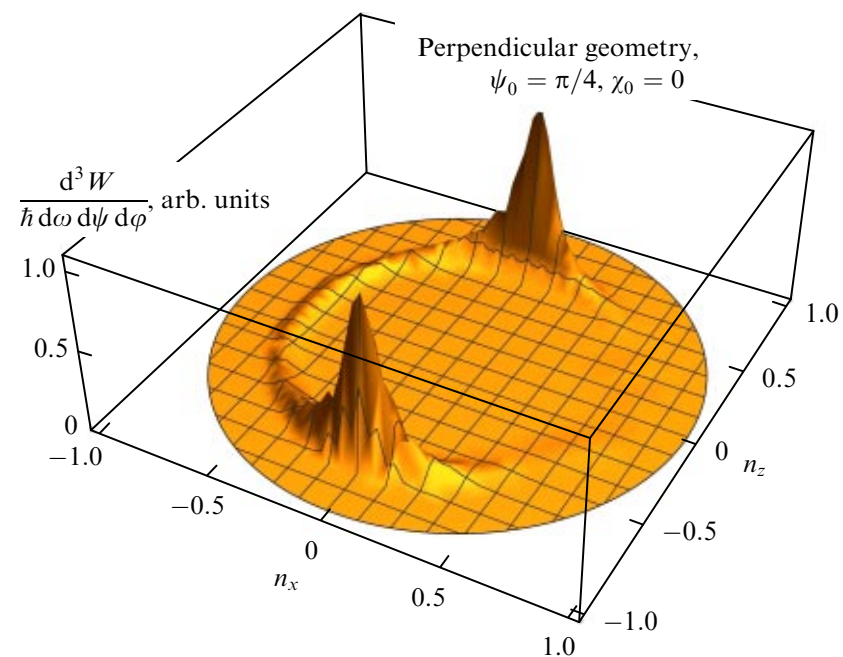

Figure 8. Angular distribution of coherent DR intensity for the perpendicular geometry and the bunch inclination angle $\chi_{0}$. The other parameters: $\gamma=10, h=1 \mathrm{~mm}, \lambda=0.5 \mathrm{~mm}, \psi_{0}=\pi / 4, \sigma_{y}=1 \mathrm{~mm}, \sigma_{z}=0.1 \mathrm{~mm}$.

For such a bunch, the velocity at which the region excited by the bunch is moving along the target edge can be easily found from geometrical considerations:

$$
\begin{aligned}
& \Delta t=\frac{2 \sigma_{y}}{\beta c}\left(\sin \chi_{0}+\frac{\cos \chi_{0}}{\tan \psi_{0}}\right) \\
& v_{\mathrm{SL}}=\frac{2 \sigma_{y} \cos \chi_{0} / \sin \psi_{0}}{\Delta t}=\beta c \frac{\cos \chi_{0}}{\cos \left(\psi_{0}-\chi_{0}\right)} .
\end{aligned}
$$

The last relationship defines the opening angle aperture of the superluminal CR cone:

$$
\cos \theta_{\mathrm{ch}}=\frac{c}{v_{\mathrm{SL}}}=\frac{\cos \left(\psi_{0}-\chi_{0}\right)}{\beta \cos \chi_{0}} .
$$

Based on the approach proposed in [7], the form factor for a bunch described by a three-dimensional Gaussian function with the parameters $\left(\sigma_{x}, \sigma_{y}, \sigma_{z}\right)$, turned over the angle $\chi_{0}$ relative to the $z^{\prime}$ axis (see Fig. 7) with phase (15), can be expressed analytically:

$$
\begin{aligned}
F(\lambda, \mathbf{n}) & =\exp \left\{-\frac{4 \pi^{2}}{\lambda^{2}}\left[\sigma_{y}^{2}\left(\sin \chi_{0}-n_{y}^{\prime} \cos \chi_{0}\right)^{2}\right.\right. \\
& \left.\left.+\sigma_{z}^{2}\left(\cos \chi_{0}+n_{y}^{\prime} \sin \chi_{0}\right)^{2}\right]\right\}
\end{aligned}
$$

In the ultra-relativistic case, provided the relationship $\sigma_{y}^{2} \gg \sigma_{z}^{2}$ is true, form factor (18) attains a maximum under the condition

$$
n_{y}^{\prime}=\frac{\sin \chi_{0}}{\cos \chi_{0}}
$$

when the first term (the main one) in the argument of the exponential function becomes zero.

For a bunch with $\chi_{0}=0$ (the minor ellipsoid axis is along the velocity), from (19) it follows that

$$
n_{y}^{\prime}=0, \text { or } \cos \psi-\frac{\cos \psi_{0}}{\beta}=0,
$$

which coincides with the condition of CR for a single charge (8), which also remains valid for a bunch in the case

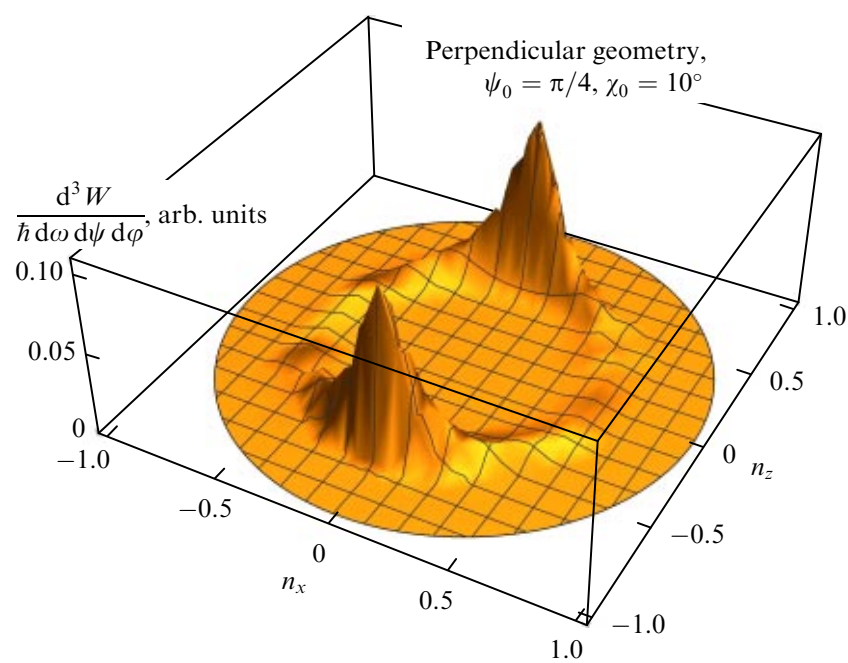

Figure 9. The same as in Fig. 8, but for the bunch inclination angle $\chi_{0}=10^{\circ}$.

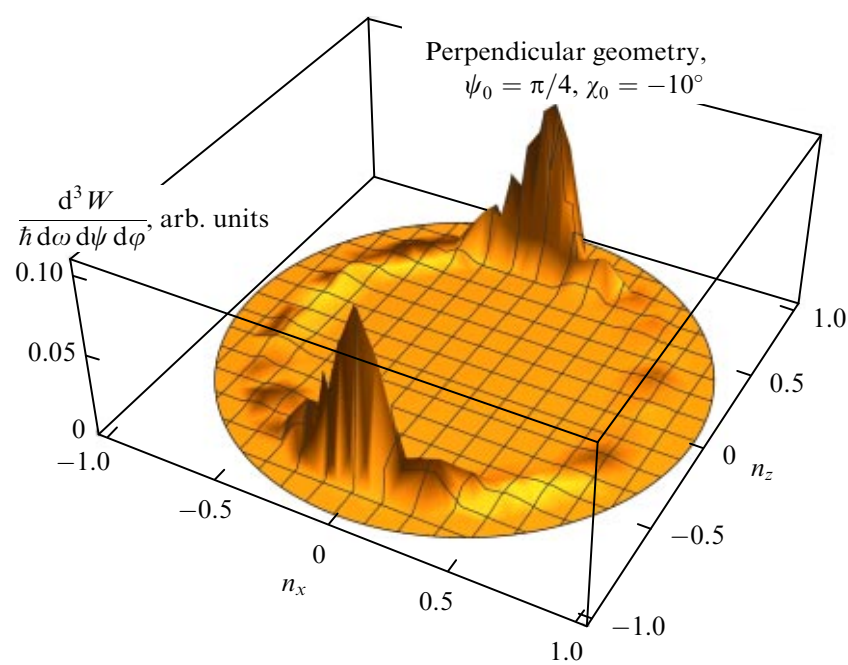

Figure 10. The same as in Fig. 9, but for the bunch inclination angle $\chi_{0}=-10^{\circ}$.

considered. In general, when $\chi_{0} \neq 0$, condition (19) reduces to the following:

$$
\cos \theta_{\mathrm{ch}}=\cos \psi_{\mathrm{m}}=\frac{\sin \chi_{0}}{\cos \chi_{0}} \sin \psi_{0}+\frac{\cos \psi_{0}}{\beta} \approx \frac{\cos \left(\psi_{0}-\chi_{0}\right)}{\beta \cos \chi_{0}}
$$

Thus, the CR cone angle changes if the bunch is oblique. For $\chi_{0}>0$, the cone becomes narrower, and it becomes wider for $\chi_{0}<0$.

Figures 8-10 depict angular distributions of coherent diffraction radiation for the perpendicular geometry, illustrating the CR cone deformation according to formula (21).

\section{Threshold condition for the occurrence of 'superluminal' Cherenkov radiation in a vacuum}

Recent paper [20] considers radiation generated by a front of a sheet electron beam as it passes over an array of holes 
oriented at an angle $\alpha$ to the electron momentum. The authors of [20] came to the conclusion that the radiation mechanism follows the Cherenkov mechanism in the zeroth order for the case they consider and obtained a formula for the Cherenkov radiation angle (see formula (3) in [20]), which, as should be expected, coincides with formulas (8) and (12) obtained by us earlier (see [14, 15]).

However, the main statement of [20], "Here we illustrated a threshold-less Cherenkov radiation (CR) in vacuo by using a sheet free-electron beam to excite an oblique-lined subwavelength hole array," is incorrect. CR, in its physical sense, presents a 'threshold effect', i.e., it arises only when the velocity of the radiation source exceeds the speed of light, and exactly for this reason relationships (8), (12), as well as formula (3) in [20], define the threshold source velocity - the effect simply does not exist for lower velocities.

The authors of [20] carried out modeling of radiation characteristics for very low electron energy $E_{\mathrm{kin}}=5 \mathrm{keV}$ and the hole array inclined at $\psi_{0}=85^{\circ}$ and demonstrated the CR yield at the angle of $\psi_{\mathrm{m}}=51^{\circ}$, in agreement with formula (8), which served as the basis of their claim that there is no threshold for the effect studied. However, for the given kinetic energy, the relative velocity of electrons imposes the 'threshold' condition on the inclination angle $\psi_{0}$ :

$$
\cos \psi_{0}<\beta \text { or } \psi_{0}>81.95^{\circ} \text {. }
$$

Thus, the threshold condition, albeit a 'weak' one, is necessarily present.

From our standpoint, the title of Ref. [20], "Thresholdless and focused Cherenkov radiation using sheet electron beams to drive sub-wavelength hole arrays," may confuse the reader. We just carried out a detailed analysis of an effect analogous to that treated in [20] and demonstrated that the claim by the authors of [20] concerning the threshold-less character of the Cherenkov mechanism is groundless.

\section{Conclusions}

We showed that the DR from a single charge as well as the coherent DR of short bunches can be treated as 'superluminal' CR emitted by the area of dynamically polarized atomic shells moving along the target edge at a superluminal speed. The problem lacks azimuthal symmetry, and the distribution of DR along the CR cone generating lines is asymmetric too.

In all known experiments which explored incoherent [2123] and coherent [24, 25] DR, the angle $\psi_{m}$ at which DR photon emission intensity is a maximum coincides with the half-angle of the CR cone $\theta_{\mathrm{ch}}$ and is defined by the component of electron velocity along the target edge (or grating strip [26]) according to formula (12b). Note that when the bunch front is oblique relative the target surface/strip line of the grating, which takes place for coherent DR of ultrashort electron bunches, $\sigma_{x, y} \gg \sigma_{z}$, the CR cone will also be formed because of the bunch inclination angle $\chi_{0}$ (see expression (21)). Characteristics of this type can be used for noninvasive diagnostics of femtosecond electron bunches. Reference [19] proposed to use coherent transition radiation with this goal, which will inevitably deteriorate the parameters of a beam passing through the target material.

The approach to the description of DR, as well as resonance DR (in particular, Smith-Purcell radiation), based on the concept of superluminal $\mathrm{CR}$, allows one to easily determine the direction of maximum radiation yield for a given geometry (the angle $\psi_{\mathrm{m}}$ ) without resorting to cumbersome process modeling, as proposed in [20].

The authors would like to use this pleasant opportunity to thank V V Syshchenko for the stimulating discussions. The work was financially supported through grant 18-52-50002/ 18 from the Russian Foundation for Basic Research.

\section{References}

1. Bolotovskii B M, Ginzburg V L Sov. Phys. Usp. 15184 (1972); Usp. Fiz. Nauk 106577 (1972)

2. Ginzburg V L Theoretical Physics and Astrophysics (Oxford: Pergamon Press, 1979); Translated from Russian: Teoreticheskaya Fizika i Astrofizika. Dopolnitel'nye Glavy (Moscow: Nauka, 1975)

3. Bolotovskii B M, Serov A V Phys. Usp. 48903 (2005); Usp. Fiz. Nauk 175943 (2005)

4. Shul'ga N F, Syshchenko V V Phys. Lett. A 313307 (2003)

5. Shul'ga N F, Syshchenko V V, Soboleva N V Poverkhnost (11) 83 (2009)

6. Bolotovskii B M, Serov A V Tech. Phys. 471 (2002); Zh. Tekh. Fiz. 72 (1) 3 (2002)

7. Potylitsyn A P JETP Lett. 103669 (2016); Pis'ma Zh. Eksp. Teor. Fiz. 103762 (2016)

8. Bolotovskii B M, Galst'yan E A Phys. Usp. 43755 (2000); Usp. Fiz. Nauk 170809 (2000)

9. Tishchenko A A, Potylitsyn A P, Strikhanov M N Phys. Rev. E 70 066501 (2004)

10. Karlovets D V, Potylitsyn A P Phys. Lett. A 3731988 (2009)

11. Barsukov K A, Popov V N Phys. Usp. 391181 (1996); Usp. Fiz. Nauk 1661245 (1996)

12. Arkhipov R M et al. Phys. Rev. A 89043811 (2014)

13. Landau L D, Lifshitz E M Electrodynamics of Continuous Media (Oxford: Pergamon Press, 1993); Translated from Russian: Elektrodinamika Sploshnykh Sred (Moscow: Nauka, 1992)

14. Sergeeva D Yu, Tishchenko A A, Strikhanov M N Phys. Rev. ST Accel. Beams 18052801 (2015)

15. Sergeeva D Yu, Tishchenko A A, Strikhanov M N Nucl. Instrum. Meth. Phys. Res. B 355155 (2015)

16. Potylitsyn A P et al. Diffraction Radiation from Relativistic Particles (Springer Tracts in Modern Physics, Vol. 239) (Heidelberg: Springer, 2010)

17. England R J et al. Rev. Mod. Phys. 861337 (2014)

18. Dorda U et al. Nucl. Instrum. Meth. Phys. Res. A 829233 (2016)

19. Tsarev M V, Baum P New J. Phys. 20033002 (2018)

20. Liu Y et al. Opt. Express 2634994 (2018)

21. Karataev P et al. Phys. Rev. Lett. 93244802 (2004)

22. Lumpkin A H et al. Phys. Rev. ST Accel. Beams 10022802 (2007)

23. Chiadroni E et al. Nucl. Instrum. Meth. Phys. Res. B 2663789 (2008)

24. Castellano M et al. Phys. Rev. E 63056501 (2001)

25. Aleinik A N et al. JETP Lett. 76337 (2002); Pis'ma Zh. Eksp. Teor. Fiz. 76397 (2002)

26. Naumenko G A et al. JETP Lett. 105553 (2017); Pis'ma Zh. Eksp. Teor. Fiz. 105531 (2017) 